\title{
Collision of Control Sets
}

Fritz Colonius ${ }^{1}$ and Wolfgang Kliemann ${ }^{2, \star}$

1 Institut für Mathematik, Universität Augsburg, 86135 Augsburg, Germany email: colonius@math.uni-augsburg.de

2 Department of Mathematics, Iowa State University, Ames, Iowa 50011, U.S.A. email: kliemann@iastate.edu

Abstract. Control sets describe the limit behavior of control systems. When they collide, the system behavior undergoes a qualitative change. It is shown that at a collision of an invariant control set $C$ with another control set $D$ there exists a $\partial C$-control set in the intersection of $C$ and the closure of $D$.

\section{Introduction}

In this paper, qualitative properties of nonlinear control systems are discussed. The methods and results are a combination of geometric control theory and topological theory of dynamical systems. This approach (combined with numerical methods) has yielded a number of new insights in the last years. The basic theory is presented in the book [2]. In the present paper we analyze the collision of control sets; in particular, we describe the intersection points of an invariant control set with the closure of another control set.

We consider control-affine systems of the form

$$
\begin{aligned}
& \dot{x}=X_{0}(x)+\sum_{i=1}^{m} u_{i}(t) X_{i}(x)=: X(x, u(t)), \\
& u \in \mathcal{U}=\left\{u \in L_{\infty}\left(\mathbb{R}, \mathbb{R}^{m}\right), u(t)=\left(u_{i}(t)\right) \in U \text { for almost all } t \in \mathbb{R}\right\},
\end{aligned}
$$

where $X_{0}, \ldots, X_{m}$ are $C^{\infty}$-vector fields on a Riemannian manifold $M$ and $U$ is a nonvoid, convex, and compact subset $U$ of $\mathbb{R}^{m}$. We assume throughout, that for every initial value $x \in X$ and every $u \in \mathcal{U}$ there exists a unique solution $\varphi(t, x, u), t \in \mathbb{R}$, with $\varphi(0, x, u)=x$.

The functions $u \in \mathcal{U}$ can be interpreted as controls, which are chosen to achieve a desired system behavior or as time-dependent perturbations acting on the system. The dynamical properties of system (1) are embodied in the associated control flow given by

$$
\Phi: \mathbb{R} \times \mathcal{U} \times M \rightarrow \mathcal{U} \times M, \Phi_{t}(u, x)=(u(t+\cdot), \varphi(t, x, u)) .
$$

Then $\Phi$ becomes a continuous dynamical system if we endow $\mathcal{U}$ with the weak $^{*}$ topology of $L_{\infty}\left(\mathbb{R}, \mathbb{R}^{m}\right)$, and $\mathcal{U} \times M$ is a metrizable space. The limit

* Project: Analysis of Time-Dependent Perturbations of Ordinary Differential Equations (Fritz Colonius) 
behavior of $\Phi$ is determined by the control sets and the chain control sets, which correspond to the topologically transitive components and the chain transitive components, respectively, of $\Phi$. Generically, they coincide (see Section 2 for a precise statement). Qualitative changes occur when an invariant, hence closed, control set intersects the closure of another control set. Then both control sets are contained in a single chain control set and we will discuss this situation assuming local accessibility. We remark that Grünvogel [7] has described when a singular point lies in the closure of a control set with nonvoid interior. His analysis of this case where local accessibility is violated is based on Lyapunov exponents.

In Section 2 we introduce some notation and recall some facts on control sets and chain control sets. Section 3 defines control sets relative to a compact subset $L$ of the state space and discusses their basic properties. Section 4 presents the main result, establishing the existence of a control set relative to the boundary $\partial C$ of an invariant control set $C$, when $C$ collides with another control set. Section 5 concentrates on the two dimensional case, based on results of Poincaré-Bendixson type. Section 6 presents some numerically computed examples.

\section{Basic Properties of Control Sets}

In this section, we cite the pertinent results from [2] on the global controllability structure of control systems.

We consider control system (1) with controls $u \in \mathcal{U}$. Sometimes it will be convenient to introduce a parameter $\rho \geq 0$, which indicates the size of the perturbations and to replace the pointwise constraint $u(t) \in U$ by

$$
u(t) \in U^{\rho}:=\rho \cdot U
$$

Then the control functions are in

$$
\mathcal{U}^{\rho}=\left\{u \in L_{\infty}\left(\mathbb{R}, \mathbb{R}^{m}\right), u(t) \in U^{\rho} \text { for almost all } t \in \mathbb{R}\right\}
$$

In particular, for $\rho=0$, one recovers the nominal, unperturbed system. If the dependence on $\rho$ does not play a role, we simply write $\mathcal{U}$. Throughout we assume that system (1) is locally accessible, i.e., for all $x \in \mathbb{R}^{d}$ and all $T>0$ one has

$$
\operatorname{int} \mathcal{O}_{\leq T}^{+}(x) \neq \emptyset \text { and } \operatorname{int} \mathcal{O}_{\leq T}^{-}(x) \neq \emptyset
$$

where $\mathcal{O}_{\leq T}^{+}(x)=\{y \in M, y=\varphi(t, x, u)$ with $0 \leq t \leq T$ and $u \in \mathcal{U}\}$ and $\mathcal{O}_{<T}^{-}(x)=\{y \in M, x=\varphi(t, y, u)$ with $0 \leq t \leq T$ and $u \in \mathcal{U}\}$ are the positive and negative reachable sets (or orbits), respectively, from $x$.

To describe the global behavior of system (1), we recall the following concepts and results. 
Definition 1. A subset $D$ of the state space $M$ is a control set if it is a maximal subset with the properties that (i) for all $x \in D$ the inclusion $D \subset$ $\operatorname{cl}^{+}(x)$ holds and (ii) for all $x \in D$ there is $u \in \mathcal{U}$ with $\varphi(t, x, u) \in D$ for all $t \geq 0$. A control set $C$ is an invariant control set if $\operatorname{cl} C=\operatorname{cl} \mathcal{O}^{+}(x)$.

If the dependence of $D$ on $\rho$ plays a role, we indicate this by the argument $\rho$ in $D(\rho)$. The same remark applies to all other notions introduced. The local accessibility assumption guarantees that the invariant control sets are the closed control sets and that they have nonvoid interior.

We will also need the following weaker concept, chain control sets, which allows for arbitrarily small jumps in the trajectories. For $\varepsilon, T>0$, a controlled $(\varepsilon, T)$-chain from $x$ to $y$ is given by $n \in \mathbb{N}$, times $T_{0}, \ldots, T_{n-1} \geq T$, points $x_{0}=x, \ldots, x_{n-1}, x_{n}=y$ in $M$, and controls $u_{0}, \ldots, u_{n-1} \in \mathcal{U}$ such that

$$
d\left(\varphi\left(T_{i}, x_{i}, u_{i}\right), x_{i+1}\right)<\varepsilon \text { for all } i=0, \ldots, n-1 .
$$

Definition 2. A chain control set $E \subset M$ is a maximal subset such that (i) for all $x, y \in E$ and all $\varepsilon, T>0$ there is a controlled $(\varepsilon, T)$-chain from $x$ to $y$; and (ii) for all $x \in E$ there is $u \in \mathcal{U}$ with $\varphi(t, x, u) \in E$ for all $t \in \mathbb{R}$.

Chain control sets are closed and every control set is contained in a chain control set. Furthermore, if two control sets $D_{1}$ and $D_{2}$ satisfy $\operatorname{cl} D_{1} \cap \operatorname{cl} D_{2} \neq$ $\emptyset$, then there is a chain control set $E$ with $D_{1} \cup D_{2} \subset E$.

To get a better understanding of the limit behavior we lift the control sets $D \subset M$ with nonvoid interior to sets in $\mathcal{U} \times M$ via

$$
\mathcal{D}=\operatorname{cl}\{(u, x) \in \mathcal{U} \times M, \varphi(t, x, u) \in \operatorname{int} D \text { for all } t \in \mathbb{R}\},
$$

where the closure is taken with respect to the weak* topology on $\mathcal{U}$ and the given topology on $M$. Observe that $\mathcal{D}$ and

$$
\{(u, x) \in \mathcal{U} \times M, \varphi(t, x, u) \in \operatorname{int} D \text { for all } t \in \mathbb{R}\}
$$

are invariant under $\Phi$ and, if $D$ is bounded in the metric given by the Riemannian structure on $M$, then $\mathcal{D}$ is compact.

Theorem 3. Let $\mathcal{D} \subset \mathcal{U} \times M$ be a set such that the projection in $M$

$$
\pi_{M} \mathcal{D}=\{x \in M, \text { there exists } u \in \mathcal{U} \text { with }(u, x) \in \mathcal{D}\}
$$

is compact. Then $\mathcal{D}$ is a maximal topologically mixing set if and only if there exists a control set $D$ such that $\mathcal{D}$ is of the form (2). In this case $D$ is unique; int $D=\operatorname{int} \pi_{M} \mathcal{D}$, and $\mathrm{cl} D=\pi_{M} \mathcal{D}$. Furthermore, the periodic points of $\Phi$ are dense in $\mathcal{D}$; the restricted flow $\Phi \mid \mathcal{D}$ is topologically mixing, topologically transitive, and chain transitive, and it has sensitive dependence on initial conditions. 
This is [2, Theorem 4.1.3]. An analogous interpretation in terms of the control flow can also be given for the chain control sets $E$. Again we consider a lift to $\mathcal{U} \times M$ by defining

$$
\mathcal{E}=\{(u, x) \in \mathcal{U} \times M, \varphi(t, x, u) \in E \text { for all } t \in \mathbb{R}\} .
$$

Theorem 4. Let $E \subset M$ be a chain control set. Then $\mathcal{E} \subset \mathcal{U} \times M$ as defined by (3) is a maximal invariant chain transitive set for the control flow $\mathcal{U} \times$ $M, \Phi)$. Conversely, let $\mathcal{E} \subset \mathcal{U} \times M$ be a maximal invariant chain transitive set for $(\mathcal{U} \times M, \Phi)$. Then $\pi_{M} \mathcal{E}$ is a chain control set.

This is [2, Theorem 4.1.4]. In general, the control sets are proper subsets of the chain control sets. Considering systems with varying control ranges $U^{\rho}$, one finds that the relation between the control sets and chain control sets above is much closer. The chain control sets coincide generically with the closures of the control sets. Indicating the $\rho$-dependence by the argument $\rho$, we have $\operatorname{cl} D(\rho) \subset E(\rho)$ for increasing families $D(\rho)$ and $E(\rho), \rho>0$. Let

$$
\mathcal{D}(\rho):=\operatorname{cl}\left\{(u, x) \in \mathcal{U}^{\rho} \times \mathbb{R}^{d}, \varphi(t, x, u) \in \operatorname{int} D(\rho) \text { for all } t \in \mathbb{R}\right\} .
$$

Theorem 5. Assume that the following inner pair condition holds: for all $\rho^{\prime}>\rho>0$ and all $(u, x) \in \mathcal{D}(\rho)$ there is $T>0$ with $\varphi(T, x, u) \in \operatorname{int} \mathcal{O}^{+, \rho^{\prime}}(x)$. Then the map $\rho \mapsto \operatorname{cl} D(\rho)$ is lower semicontinuous and the map $\rho \mapsto E(\rho)$ is upper semicontinuous (with respect to Hausdorff metric). The sets of continuity points for both maps coincide, and $\rho^{*} \in(0, \infty)$ is a continuity point if and only if $\mathrm{cl} D\left(\rho^{*}\right)=E\left(\rho^{*}\right)$. There are at most countably many point of discontinuity.

This theorem (see [2, Theorem 4.7.5]) shows that under the inner pair condition the chain control sets 'almost always' coincide with the closures of control sets. The exceptional cases will be of particular interest. We remark that the inner pair condition can be verified for a number of second order systems with additive controls; compare [4].

\section{$3 \quad L$-Control Sets}

In this section, we describe control sets which are defined relative to a subset $L$ of the state space.

Let $L \subset M$ be a subset such that every point of $L$ is a cluster point of $L$, i.e., for every $x \in L$ there are $x_{n} \in L$ with $x \neq x_{n} \rightarrow x$. Furthermore we assume that $L$ is positively viable (or controlled invariant), i.e., for every $x \in L$ there is $u \in \mathcal{U}$ such that $\varphi(t, x, u) \in L$ for all $t \geq 0$.

Definition 6. For $x \in L$, the positive $L$-orbit of $x$ is given by

$$
\mathcal{O}_{L}^{+}(x)=\left\{y \in L, \begin{array}{c}
\text { there are } t>0 \text { and } u \\
\text { and } \varphi(s, x, u) \in L \text { such that } \varphi(t, x, u)=y \\
\text { all } 0<s<t
\end{array}\right\} .
$$

Analogously, one defines $\mathcal{O}_{L, \leq t}^{+}(x), \mathcal{O}_{L}^{-}(x), \mathcal{O}_{L, \leq t}^{-}(x)$, etc. 
Definition 7. An $L$-control set $F$ is a subset of $L$ with the following properties:

(i) for all $x \in F$ the inclusion $F \subset \operatorname{cl} \mathcal{O}_{L}(x)$ holds, (ii) for all $x \in F$ there is $u \in \mathcal{U}$ with $\varphi(t, x, u) \in F$ for all $t \geq 0$, and (iii) the set $F$ is maximal with these properties. An $L$-control set $F$ is an invariant $L$-control set if

$$
\mathrm{cl}_{L} F=\operatorname{cl}_{L} \mathcal{O}_{L}^{+}(x),
$$

where the closures are taken relative to the set $L$.

Thus an invariant $L$-control set cannot be left without leaving $L$.

Definition 8. The system (1) is called $L$-accessible if for all $x \in L$ and all $t>0$ the sets $\mathcal{O}_{L, \leq t}^{+}(x)$ and $\mathcal{O}_{L, \leq t}^{-}(x)$ have nonvoid interiors relative to $L$.

For $L=M$, these definitions reduce to the standard definitions recalled earlier. We start with the following simple observation.

Proposition 9. Let $D$ be a control set with nonvoid interior. Then int $D=$ int $\mathrm{cl} D$ and this set is path connected.

Proof. Let $x \in \operatorname{int} \operatorname{cl} D$. By local accessibility, $\emptyset \neq \mathcal{O}_{\leq t}^{ \pm}(x) \subset \operatorname{int} \operatorname{cl} D$ for $t>0$, small enough. Hence, using continuous dependence on the initial value, one finds that $x \in \operatorname{int} D$. The converse inclusion is clear. Path connectedness follows from exact controllability in int $D$.

Proposition 10. Let $D$ be a control set with nonvoid interior. Then every point of the boundary $\partial D$ is a clusterpoint of $\partial D$ and $\partial D$ is viable, i.e., for every $x \in \partial D$ there exists $u \in \mathcal{U}$ such that $\varphi(t, x, u) \in \partial D$ for all $t \in \mathbb{R}$.

Proof. The first assertion follows, because int $D=\operatorname{int} \operatorname{cl} D$. For the second assertion, fix $x \in \partial D$ : Then there are $y \in \operatorname{int} D$ and $v \in \mathcal{U}$ with $\varphi\left(t_{n}, y, v\right) \rightarrow x$ for some $t_{n} \rightarrow \infty$. Take a clusterpoint $u_{-1} \in \mathcal{U}$ of the sequence $\left(v\left(t_{n}+\cdot\right)\right)$. Then $\varphi\left(-1, x, u_{-1}\right) \in \partial D$, since it is in $\operatorname{cl} D$ (because it is a clusterpoint of $\left.\varphi\left(t_{n}-1, y, v\right) \in \operatorname{int} D\right)$; and it is not in int $D$, since the boundary point $x$ cannot be reached in finite time from the interior of $D$. Proceeding in this way, one constructs a control $u_{-} \in \mathcal{U}$ with $\varphi\left(t, x, u_{-}\right) \in \partial D$ for all $t \leq 0$. Applying the same procedure to the time reversed system, one finds a control $u_{+} \in \mathcal{U}$ with $\varphi\left(t, x, u_{+}\right) \in \partial D$ for all $t \geq 0$. Thus $u(t)=u_{ \pm}(t)$ for $t \leq 0$ and $t \geq 0$, respectively, has the desired property.

Proposition 11. Suppose that $D$ is a control set with nonvoid interior and compact closure. Then for every $x \in \partial D$ there exists an invariant $\partial D$-control set $F \subset \operatorname{cl} \mathcal{O}_{\partial D}^{+}(x)$. 
Proof. By Proposition 10 it follows that for every $x \in \partial D$ the positive $\partial D$ orbit

$$
\mathcal{O}_{\partial D}^{+}(x)=\left\{y \in \partial D, \begin{array}{c}
\text { there are } t>0 \text { and } u \in \mathcal{U} \text { such that } \varphi(t, x, u)=y \\
\text { and } \varphi(s, x, u) \in \partial D \text { for all } 0<s<t
\end{array}\right\}
$$

is nonvoid. Now we mimic the proof for the existence of invariant control sets in compact forward invariant sets; $\mathrm{cp}$. [2, Theorem 3.2.8]. By compactness of $\operatorname{cl} D$ the boundary $\partial D$ is compact. The family

$$
\mathcal{F}=\left\{\operatorname{clO}_{\partial D}^{+}(x), x \in \partial D\right\}
$$

is nonvoid and partially ordered by set inclusion. By compactness of $\partial D$, every linearly ordered subset has a lower bound. Hence $\mathcal{F}$ satisfies the assumptions of Zorn's lemma, yielding existence of a minimal element, which is an invariant $\partial D$-control set. Furthermore, for every $x \in \partial D$ there is an invariant $\partial D$-control set in $\operatorname{cl}_{\partial D}^{+}(x)$.

Proposition 12. Suppose that the following local $\partial D$-accessibility condition holds:

$$
\operatorname{int}_{\partial D} \mathcal{O}_{\partial D, \leq t}^{ \pm}(x) \neq \emptyset \text { for all } x \in \partial D \text { and all } t>0 .
$$

Then every invariant $\partial D$-control set has nonvoid interior relative to $\partial D$. Furthermore, for every $\partial D$-control set $F$ with nonvoid interior $\operatorname{int}_{\partial D} F$ one has $\mathrm{cl} \operatorname{int}_{\partial D} F=\mathrm{cl} F$, and for all $x, y \in \operatorname{int}_{\partial D} F$ there are $t>0$ and $u \in \mathcal{U}$ with $\varphi(t, x, u)=y$.

Proof. This follows as the corresponding assertions for control sets; compare [2, Section 3.2].

\section{Collision of Control Sets}

In this section, we discuss collisions of an invariant control set $C$ with another control set $D$, i.e., $C \cap \operatorname{cl} D \neq \emptyset$. Note that then $C \cap \operatorname{cl} D=\partial C \cap \partial D$. We will show that in this case the set of contact points, i.e., the points in the intersection of $C$ with $\mathrm{cl} D$, must contain a control set relative to $\partial C$. The first result shows a weaker property.

Theorem 13. Let $C$ be a compact invariant control set for system (1). Suppose that $C \cap \operatorname{cl} D \neq \emptyset$ for some control set $D \neq C$ with nonvoid interior. Then there exist an invariant $\partial D$-control set $F^{\prime}$ and a $\partial C$-control set $F$ with

$$
F^{\prime} \subset C \cap \operatorname{cl} D \text { and } F \cap C \cap \operatorname{cl} D \neq \emptyset \text {. }
$$

Proof. Fix $x \in C \cap \mathrm{cl} D$. By Proposition 11, there exists an invariant $\partial D$ control set $F^{\prime} \subset \operatorname{cl} \mathcal{O}_{\partial D}^{+}(x)$. By invariance of $C$, it follows that $F^{\prime} \subset C \cap \operatorname{cl} D$. Then the definitions immediately imply that there is a $\partial C$-control set $F$ containing $F^{\prime}$, and the second assertion follows. 
Requiring local accessibility properties on the boundaries of the considered control sets, we obtain the following stronger result. It shows that collisions of an invariant control set with another control set occur at $\partial C$-control sets.

Theorem 14. Let $C$ be a compact invariant control set for system (1). Suppose that $C \cap \mathrm{cl} D \neq \emptyset$ for some control set $D \neq C$ with nonvoid interior.

(i) If local $\partial D$-accessibility holds, then there exist an invariant $\partial D$-control set $F^{\prime}$ with $F^{\prime} \subset \partial C \cap \partial D$ and $\operatorname{int}_{\partial D} F^{\prime} \neq \emptyset$.

(ii) If, additionally, local $\partial C$-accessibility holds and $\operatorname{int}_{\partial C}(\partial C \cap \partial D)=$ $\operatorname{int}_{\partial D}(\partial C \cap \partial D)$, then there exists a $\partial C$-control set $F$ with $\operatorname{int}_{\partial C} F \neq \emptyset$ and

$$
F \subset \partial C \cap \partial D
$$

Proof. Assertion (i) is immediate from Theorem 13 and $\emptyset \neq \operatorname{int}_{\partial D} \mathcal{O}_{\partial D, \leq t}^{+} \subset$ $F^{\prime}$. To see assertion (ii) observe that by Theorem 13 there exists a $\partial C$-control set $F \supset F^{\prime}$. The assumption $\operatorname{int}_{\partial C}(\partial C \cap \partial D)=\operatorname{int}_{\partial D}(\partial C \cap \partial D)$ implies that $\emptyset \neq \operatorname{int}_{\partial D} F^{\prime}=\operatorname{int}_{\partial C} F^{\prime} \subset \operatorname{int}_{\partial C}(F \cap \partial C \cap \partial D)$. Hence $\emptyset \neq \operatorname{int}_{\partial C} F^{\prime} \subset \operatorname{int}_{\partial C} F$ and thus $F$ is a $\partial C$-control set with nonvoid interior in $\partial C$. By Proposition 12 this implies that $\operatorname{clint}_{\partial C} F=\operatorname{cl} F$. It remains to show that

$$
\operatorname{int}_{\partial C} F \subset \partial C \cap \partial D \text {. }
$$

By Proposition 12 exact controllability in int ${ }_{\partial C} F$ holds and we know that $\emptyset \neq$ $\operatorname{int}_{\partial C} F^{\prime} \cap \partial D \cap \partial C \subset \operatorname{int}_{\partial C} F \cap \partial D \cap \partial C$. Now take $x \in \operatorname{int}_{\partial C} F^{\prime} \cap \partial D \cap \partial C$ and $y \in \operatorname{int}_{\partial C} F \cap \partial D \cap \partial C$. Then, by continuous dependence on the initial value and controllability from $x$ to $y$, the point $y$ can approximately be reached from int $D$. Conversely, one can steer the system from some point in every neighborhood of $y$ into the interior of $D$, because one can steer the system from $y$ to $x$ and from some point of every neighborhood of $x$ into the interior of $D$.

Remark 15. In [3] the invariance radius $r_{i n v}$ of control sets is discussed which indicates when a control set around an stable equilibrium of the unperturbed system loses its invariance property for $\rho>r_{i n v}$. This happens when for $\rho>r_{i n v}$ the invariant control set merges with another control set $D$. Although this does not imply that at the invariance radius $r_{i n v}$ the invariant control set has nonvoid intersection with the closure of another control set $D$, this is what we see in a number of examples. In this case, the theorems above give results on the intersection of $C$ and $\operatorname{cl} D$.

\section{Poincaré-Bendixson Theory}

In this section we will sketch some results of Poincaré-Bendixson type for control systems by discussing $\omega$-limit sets for two-dimensional systems. These constructions are a modification of those in Colonius and Sieveking [5], where 
the limit behavior of optimal solutions in discounted optimal control problems is discussed. Then we use these results to describe $\partial D$-control sets in dimension $d=2$.

First we need generalizations of transversal sections and flow boxes.

Definition 16. Let $x^{0} \in \mathbb{R}^{d}, l: \mathbb{R}^{d} \rightarrow \mathbb{R}$ linear, and $\alpha>0$. If $l X(x, u)>0$ for all $x$ in a neighborhood $W$ of $x^{0}$ and all $u \in U$, then $S:=W \cap l^{-1}\left(x^{0}\right)$ is a local transversal section through $x^{0}$.

We note the following observations.

Lemma 17. (i) Suppose that $0 \notin X\left(x^{0}, U\right)$. Then $x^{0}$ has a local transversal section.

(ii) A subset $L \subset \mathbb{R}^{d}$ either contains an equilibrium or every point in $L$ has a transversal section.

Proof. Assertion (i) follows from the Hahn-Banach theorem, because $X\left(x^{0}, U\right)$ is compact and convex, and hence can be separated from $0 \notin X\left(x^{0}, U\right)$. Assertion (ii) is immediate from (i), because $L$ either contains a point $x^{0}$ with $0 \in X\left(x^{0}, U\right)$ (i.e., an equilibrium) or $0 \notin X\left(x^{0}, U\right)$ for all $x^{0} \in L$.

Definition 18. Let $S$ be a local transversal section through $x^{0}$, and let $V_{1} \subset$ $V_{0}$ be neighborhoods of $x^{0}$. Then the triple $\left(V_{0}, V_{1}, S\right)$ is called a flow box around $x^{0}$, if it has the following property: If $\varphi\left(\cdot, x^{0}, u\right)$ satisfies

$\varphi\left(t_{0}, x^{0}, u\right) \notin V_{0}, \varphi\left(t_{1}, x^{0}, u\right) \in V_{1}, \varphi\left(t_{2}, x^{0}, u\right) \notin V_{0}$ for some $0 \leq t_{0}<t_{1}<t_{2}$,

then there exists $t \in\left(t_{0}, t_{2}\right)$ such that $\varphi\left(t, x^{0}, u\right) \in S$ and $\varphi\left(s, x^{0}, u\right) \in V_{0}$ for all $s$ between $t$ and $t_{1}$.

Theorem 19. Let $S$ be a local transversal section through $x^{0}$. Then there are neighborhoods $V_{0}$ and $V_{1}$ of $x^{0}$ such that $\left(V_{0}, V_{1}, S\right)$ is a flow box around $x^{0}$.

Proof. This is [5, Theorem 2.16].

We cite Jordan's curve theorem, see, e.g., Beck [1, Corollary C.23].

Theorem 20. Let $J$ be a Jordan curve in $\mathbb{R}^{2}$. Then $\mathbb{R}^{2} \backslash J$ has two components, one of which is bounded (called ins $J$ ) and the other one (called outs $J$ ) is unbounded. Both have boundary im $J$ and are simply connected.

Since the orientation does not concern us, we identify $J$ with its image. Next we discuss limit sets as time tends to infinity. For $(u, x) \in \mathcal{U} \times M$ let

$$
\omega(u, x)=\left\{(v, y) \in \mathcal{U} \times M,\left(u\left(t_{k}+\cdot\right), \varphi\left(t_{k}, x, u\right)\right) \rightarrow(v, y) \text { for some } t_{k} \rightarrow \infty\right\},
$$

$\pi_{2} \omega(u, x)=\{y \in M$, there is $v \in \mathcal{U}$ with $(v, y) \in \omega(u, x)\}$.

If the trajectory $\varphi(\cdot, x, u)$ is bounded for positive time, then these sets are nonvoid, compact, and connected. 
Lemma 21. Let $(u, x) \in \mathcal{U} \times \mathbb{R}^{2}$ and suppose that the corresponding trajectory $\varphi(\cdot, x, u)$ is bounded and nonself-intersecting, i.e., there do not exist $T_{2}>T_{1} \geq 0$ with $\varphi\left(T_{2}, x, u\right)=\varphi\left(T_{1}, x, u\right)$. Then a local transversal section $S$ has at most one point in common with $\pi_{2} \omega(u, x)$.

Proof. This follows as [5, Lemma 4.2].

The same arguments apply to $\alpha$-limit sets, which are obtained for time $t$ tending to $-\infty$.

Proposition 22. Let $(u, x) \in \mathcal{U} \times \mathbb{R}^{2}$ and suppose that the corresponding trajectory $\varphi(\cdot, x, u)$ is bounded and nonself-intersecting. Let $(v, y) \in \omega(u, x)$. Then $\pi_{2} \omega(v, y)$ and $\pi_{2} \alpha(v, y)$ consist of equilibria only or $\varphi(\cdot, y, v)$ intersects itself in a point $z$ which has a transversal section.

Proof. This follows as [5, Proposition 4.2].

Proposition 23. Let $(u, x) \in \mathcal{U} \times \mathbb{R}^{2}$ and assume that the corresponding trajectory $\varphi(\cdot, x, u)$ is nonself-intersecting. Suppose that there are $(v, y) \in$ $\omega(u, x)$ and $T_{2}>T_{1} \geq 0$ with $\varphi\left(T_{2}, x, u\right)=\varphi\left(T_{1}, x, u\right)$ such that this point has a local transversal section. Then

$$
\pi_{2} \omega(u, x)=\varphi\left(\left[T_{1}, T_{2}\right], y, v\right)
$$

Proof. This follows as [5, Proposition 4.5].

The announced result of Poincaré-Bendixson type is presented in the following theorem.

Theorem 24. Let $(u, x) \in \mathcal{U} \times \mathbb{R}^{2}$ with nonself-intersecting trajectory $\varphi(\cdot, x, u)$ and suppose that $\pi_{1} \omega(u, x)$ contains at most finitely many equilibria. Then one of the following cases occurs:

(i) There are $T>0$ and $(v, y) \in \omega(u, x)$ such that

$$
y=\varphi(T, y, v) \text { and } \pi_{1} \omega(u, x)=\varphi([0, T], y, v) .
$$

(ii) There are $\left(v_{i}, y_{i}\right) \in \pi_{1} \omega(u, x)$ and equilibria $e_{i}^{ \pm}$such that for all $i$

$$
\begin{aligned}
e_{i}^{-} & =\lim _{t \rightarrow-\infty} \varphi\left(t, y_{i}, v_{i}\right), e_{i}^{+}=\lim _{t \rightarrow \infty} \varphi\left(t, y_{i}, v_{i}\right), \\
\pi_{1} \omega(u, x) & =\bigcup_{i} \varphi\left(\mathbb{R}, y_{i}, v_{i}\right) \cup \bigcup\left\{e_{i}^{-}, e_{i}^{+}\right\} .
\end{aligned}
$$

Proof. This follows as [5, Theorem 4.6].

Next we will apply this result to describe boundaries of control sets. The following control sets play an exceptional role. 


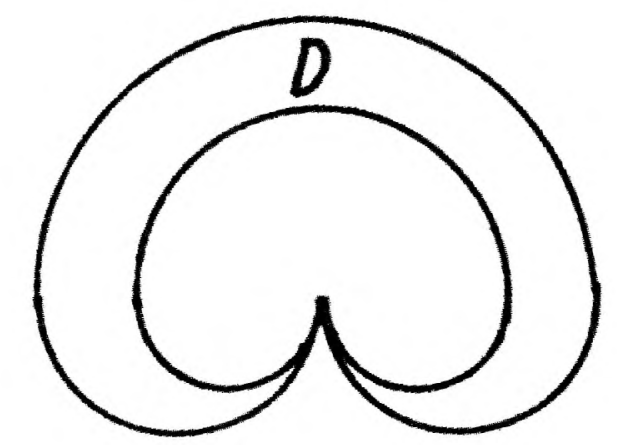

Fig. 1. Sketch of a pinched control set.

Definition 25. A control set $D \subset \mathbb{R}^{2}$ is pinched at a point $x \in \partial D$ if for every open neighborhood $W$ of $x$ the set $\partial D \cap W$ is not homeomorphic to the interval $[0,1]$.

Although it seems difficult to give an explicit example of a pinched control set, Figure 1 gives a sketch of such a control set. The next theorem describes the $\partial D$-control sets for control sets that are not pinched.

Theorem 26. Let $F$ be a $\partial D$-control set of a bounded control set $D \subset \mathbb{R}^{2}$, which is not pinched. Suppose that local $\partial D$-accessibility holds and that $F$ contains at most finitely many equilibria. Then $F$ is a single periodic trajectory, i.e., there are $T>0$ and $(v, y) \in \mathcal{U} \times \mathbb{R}^{2}$ such that $y=\varphi(T, y, v)$ and $F=\varphi([0, T], y, v)$.

Proof. Local $\partial D$-accessibility implies that $F=\pi_{2} \omega(u, x)$ for some $(u, x) \in$ $\mathcal{U} \times \partial D$. Suppose first that $\varphi(\cdot, x, u)$ is self-intersecting. Either there is $T>0$ such that $\varphi(t, x, u)=\varphi(T, x, u)$ for all $t \geq T$ and the assertion follows; or there are $T_{2}>T_{1} \geq 0$ with $\varphi\left(T_{1}, x, u\right)=\varphi\left(T_{2}, x, u\right)$ and there is $t_{0} \in\left(T_{1}, T_{2}\right)$ with $\varphi\left(t_{0}, x, u\right) \neq \varphi\left(T_{1}, x, u\right)$. If $\varphi(t, x, u)=\varphi\left(T_{1}, x, u\right)$ for some $t \in\left[T_{1}, T_{2}\right]$, then we can modify $u$ such that the new control $u^{\prime}$ yields the same set of points $\{\varphi(t, x, u), t \geq 0\}$ and $\varphi\left(t, x, u^{\prime}\right) \neq \varphi\left(T_{1}, x, u\right)$ for all $t \in\left[T_{1}, T_{2}\right]$. Then one sees that for no neighborhood $W$ of $\varphi\left(T_{1}, x, u\right)$ the set $W \cap \partial D$ is homeomorphic to $(0,1)$. It follows that the control set $D$ is pinched at the point $\varphi\left(T_{1}, x, u\right)$, contrary to our assumption. Hence $\varphi(\cdot, x, u)$ is nonselfintersecting. Then, according to Theorem 24 , one has (i) $\pi_{2} \omega(u, x)$ is a single periodic trajectory; or (ii) there are $\left(v_{i}, y_{i}\right) \in \omega(u, x)$ and equilibria $e_{i}^{ \pm}$such that for all $i$

$$
\begin{aligned}
e_{i}^{-} & =\lim _{t \rightarrow-\infty} \varphi\left(t, y_{i}, v_{i}\right), e_{i}^{+}=\lim _{t \rightarrow \infty} \varphi\left(t, y_{i}, v_{i}\right), \\
\pi_{2} \omega(u, x) & =\bigcup_{i} \varphi\left(\mathbb{R}, y_{i}, v_{i}\right) \cup \bigcup_{i}\left\{e_{i}^{-}, e_{i}^{+}\right\}
\end{aligned}
$$

In case (i) the assertion follows. In case (ii) we fix one of the equilibria $e_{i}^{ \pm}$say, $e_{1}^{-}$. Let $W$ be a neighborhood of $e_{1}^{-}$such that $\partial D \cap W$ is homeomorphic to the 
interval $(0,1)$. Then there are $u \in \mathcal{U}$ and $t_{+}, \tau_{+}>0$ such that $\varphi\left(t_{+}, e_{1}^{-}, u\right)=$ $\varphi\left(-\tau_{+}, y_{1}, v_{1}\right)$ and $\left\{\varphi\left(t, e_{1}^{+}, v_{1}\right), t \in\left[0, t_{+}\right]\right\}=\left\{\varphi\left(t, y_{1}, v_{1}\right), t \leq \tau_{+}\right\}$. Arguing analogously for all $i$ one finds that $\pi_{2} \omega(u, x)$ consists of a single periodic trajectory. This proves the assertion.

\section{Examples}

In this section we discuss control sets in a simple two-dimensional model of a continuous flow stirred tank reactor (compare Poore [8] or Golubitskii/Schaeffer [6] and also the analysis in [2, Chapter 9]). The control sets in Figures 2-5 were computed with G. Häckl's program CS.

Consider the model of a continuous flow stirred tank reactor (for short: CSTR) given by the equations

$$
\left(\begin{array}{l}
\dot{x}_{1} \\
\dot{x}_{2}
\end{array}\right)=\left(\begin{array}{l}
-x_{1}-a\left(x_{1}-x_{c}\right)+B \alpha\left(1-x_{2}\right) e^{x_{1}} \\
-x_{2}+\alpha\left(1-x_{2}\right) e^{x_{1}}
\end{array}\right)+u(t)\left(\begin{array}{c}
x_{c}-x_{1} \\
0
\end{array}\right)
$$

Here $x_{1}$ is the (dimensionless) temperature, $x_{2}$ is the product concentration, and $a, \alpha, B, x_{c}$ are positive constants. The parameter $x_{c}$ is the coolant temperature, and hence the perturbation affects the heat transfer coefficient. In [8] Poore analyzes the bifurcation behavior of the nominal, i.e., $u(t) \equiv 0$, system. Here we choose parameter values such that for all constant controls $u(t) \equiv u \in[-\rho, \rho]$ the system (4) has exactly three fixed points as limit sets. Specifically, we take for our numerical analysis

$$
a=0.15, \alpha=0.05, B=7.0, x_{c}=1.0 .
$$

First let $U=[-0.15,0.15]$. Because of the physical constraints we have to consider the system in the set $M=[0, \infty) \times[0,1] \subset \mathbb{R}^{2}$. For each fixed $u \in U$ the equation (4) has three fixed points in $M$. Let $y^{i}=\alpha e^{z^{i}} /\left(1+\alpha z^{i}\right), i=$ $0,1,2$, and let $z^{0}<z^{1}<z^{2}$ be the zeros of the transcendental equation

$$
-z-(a+u)\left(z-x_{c}\right)+B \alpha\left[1-\frac{\alpha e^{z}}{1+\alpha e^{z}}\right] e^{z}=0 .
$$

Then two of these fixed points are asymptotically stable, $x^{0}=\left(z^{0}, y^{0}\right)$ and $x^{2}=\left(z^{2}, y^{2}\right)$, and one is hyperbolic, $x^{1}=\left(z^{1}, y^{1}\right)$, i.e., the linearization about $x^{1}$ has one negative and one positive eigenvalue. The phase portrait of the nominal equation is indicated in Figure 2. There are exactly three control sets $C_{1}, C_{2}$, and $D$, containing the fixed points $x^{i}(u), i=0,1,2$, for $u \in \operatorname{int} U=(-0.15,0.15)$ in their interior. The control sets $C_{1}$ and $C_{2}$ are invariant, the control set $D$ is variant. The closures of these control sets are the three chain control sets of the system. Figure 2 shows the three control sets.

For different parameter values, Figure 3 shows the invariant control set $C_{2}$ as well as the positive and negative orbits from the hyperbolic equilibrium. 


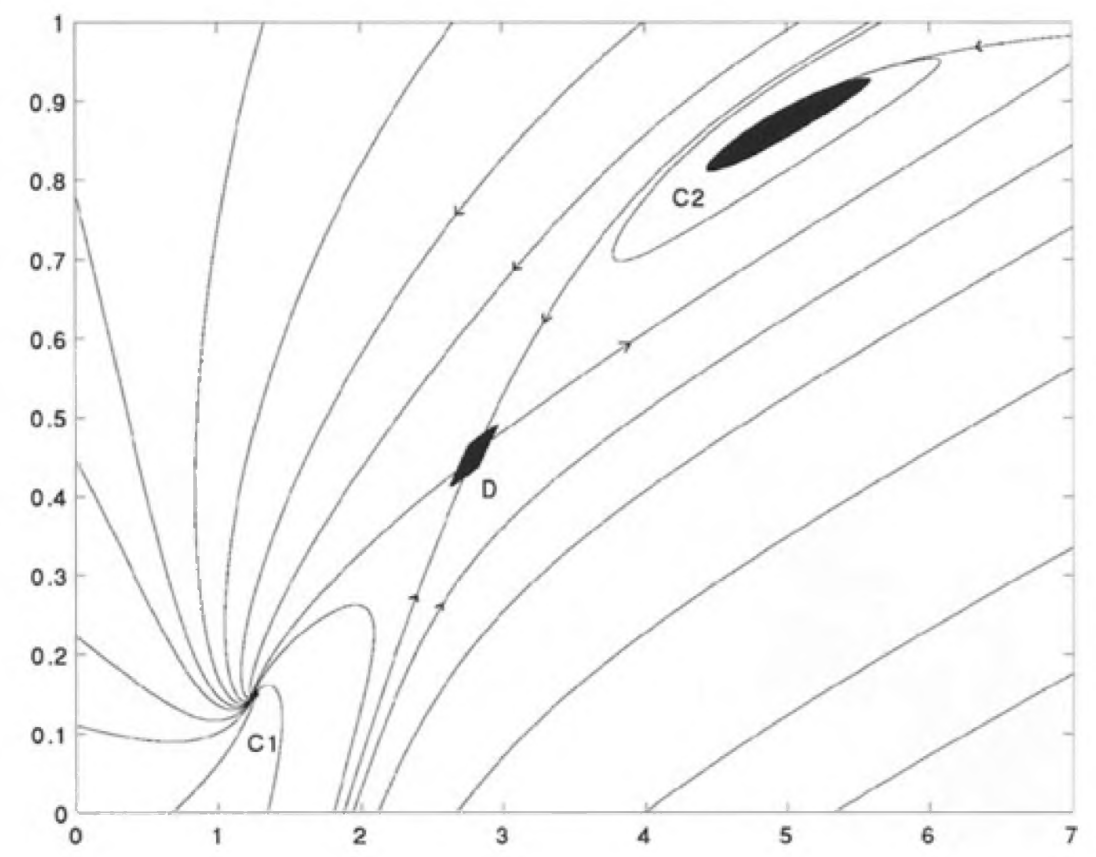

Fig. 2. Phase portrait of the unperturbed $(u(t) \equiv 0)$ continuous flow stirred tank reactor and the control sets.

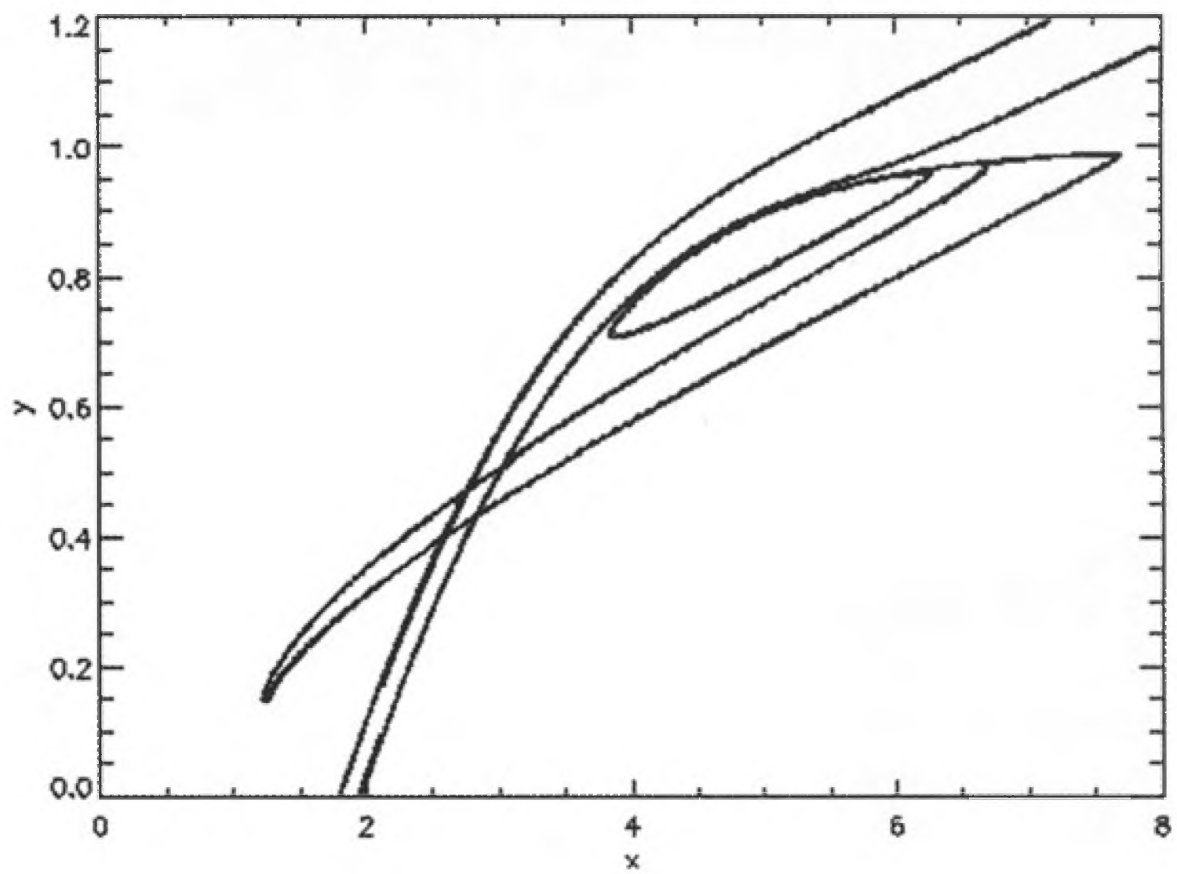

Fig. 3. Variant and invariant control sets and their domains of attraction for the CSTR 


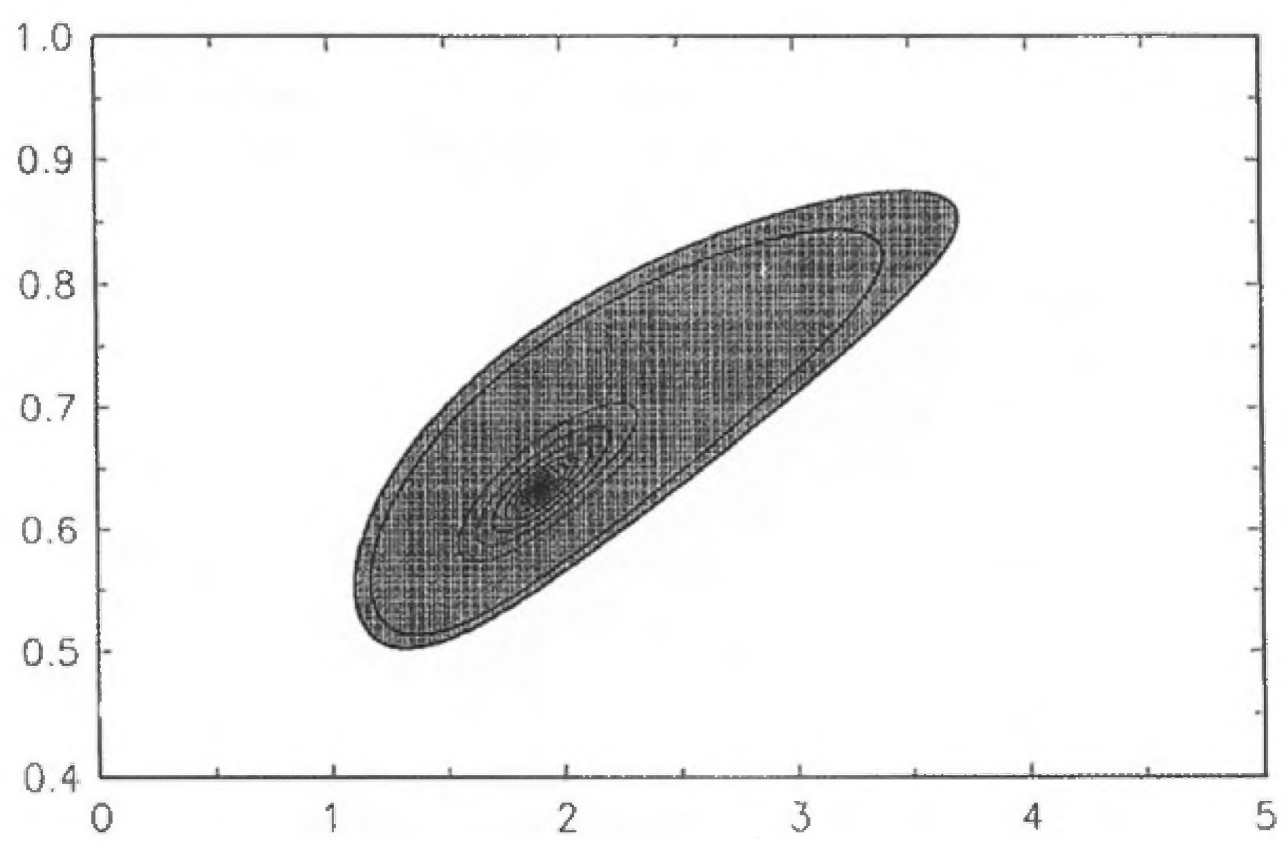

Fig. 4. Invariant control set for the CSTR with $\alpha=0.2824$, after the Hopf bifurcation.

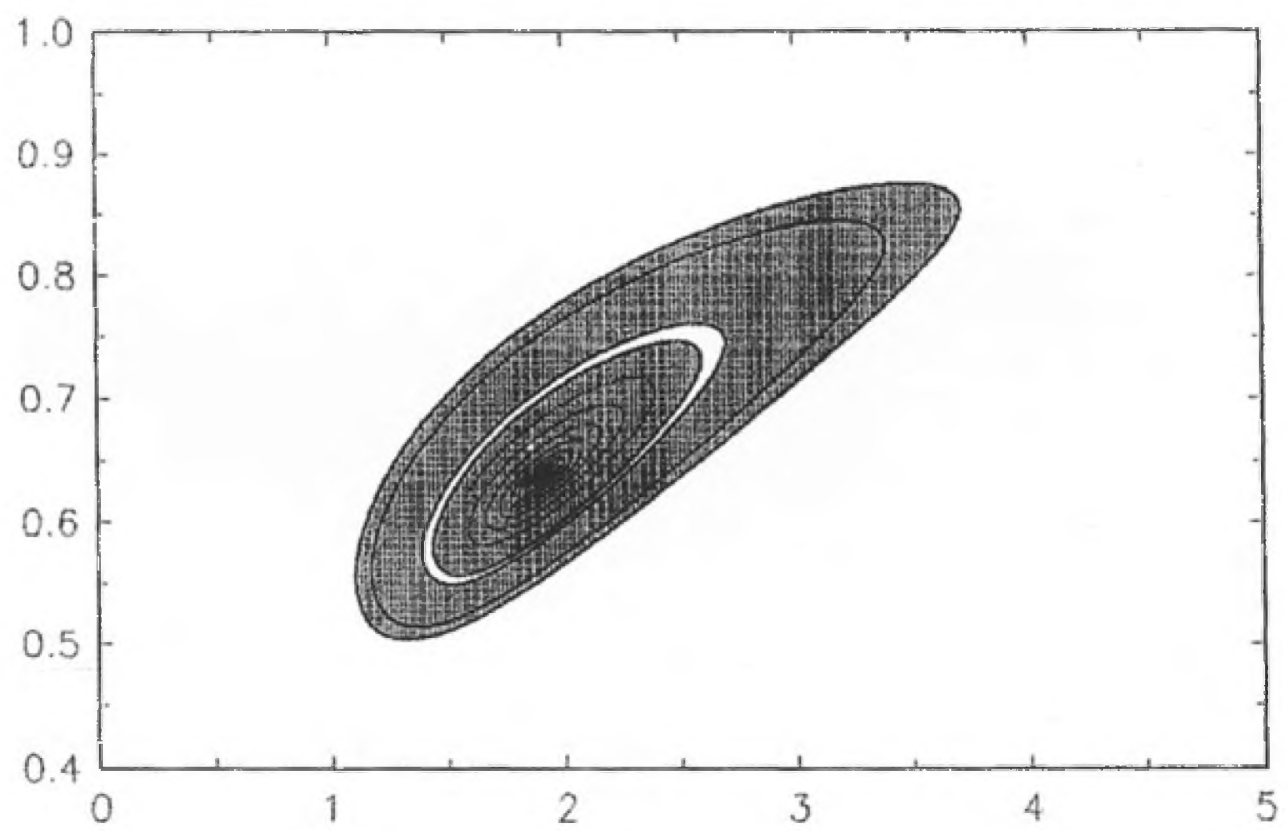

Fig. 5. Variant and invariant control sets for the CSTR with $\alpha=0.2628$. 
Their intersection is the variant control set $D$. Though the numerics seem to indicate that for a slightly larger control range the invariant control set $C_{2}$ loses its invariance by intersecting the domain of attraction $\mathbf{A}(D) \backslash \mathrm{cl} D$, the situation is different: The relevant part of the boundary of $\mathbf{A}(D)$ is the stable manifold of a hyperbolic equilibrium in $\mathrm{cl} D$. Hence, if $\mathbf{A}(D) \cap C_{2} \neq \emptyset$, it follows from invariance of $C_{2}$ that also this hyperbolic equilibrium is in $C_{2}$. The control set $C_{2}$ touches the variant control set at the hyperbolic equilibrium corresponding to the control $u=\rho$. This one-point set is an invariant $\partial C$ control set. Note that this holds, although the $\partial C$-local accessibility condition is not satisfied here.

For different parameter values, a Hopf bifurcation (taking $\alpha$ as bifurcation parameter) occurs around the upper equilibrium $x^{2}$. It loses its stability and a stable periodic solution bifurcates. Figures 4 and 5 illustrate the behavior for a fixed perturbation range with varying $\alpha$. Before and slightly after the Hopf bifurcation point there is only one invariant control set; see Figure 4. Then it bifurcates into an invariant control set around the stable periodic solution and a variant control set containing the equilibrium; see Figure 5. At the collision the invariant control set around the stable periodic solution touches the variant control set around the unstable equilibrium at the periodic solution which forms the inner boundary of the invariant control set (and coincides with the boundary of the variant control set).

\section{References}

1. A. Beck, Continuous Flows in the Plane, Springer-Verlag, 1974.

2. F. Colonius and W. Kliemann, The Dynamics of Control, Birkhäuser, 2000.

3. — An invariance radius for nonlinear systems, in Advances in Mathematical Systems. A Volume in Honor of D. Hinrichsen., F. Colonius, U. Helmke, D. Prätzel-Wolters, and F. Wirth, eds., Birkhäuser, 2000.

4. - Invariance under bounded time-varying perturbations, in 11th IFAC International Workshop Control Applications of Optimization (St. Petersburg, Russia, July 3-6 2000), V. Zakharov, ed., 2000, pp. 82-85.

5. F. Colonius and M. Sievering, Asymptotic properties of optimal solutions in planar discounted control problems, SIAM J. Control Optim., 27 (1989), pp. 608 630.

6. M. Golubitsky and D. Schaeffer, Singularities and Groups in Bifurcation Theory, Springer-Verlag, 1985.

7. S. Grünvogel, Control Sets and Lyapunov Spectrum. Dissertation, Universität Augsburg, 2000.

8. A. B. Poore, A model equation arising from chemical reactor theory, Arch. Rational Mech. Anal., 52 (1974), pp. 358-388. 